\title{
Positive Solutions of a Nonlinear Parabolic Partial Differential Equation
}

\author{
Chengbo Zhai and Shunyong Li \\ School of Mathematical Sciences, Shanxi University, Taiyuan, Shanxi 030006, China \\ Correspondence should be addressed to Chengbo Zhai; cbzhai@sxu.edu.cn
}

Received 13 March 2014; Accepted 19 May 2014; Published 28 May 2014

Academic Editor: Dragos-Patru Covei

Copyright (C) 2014 C. Zhai and S. Li. This is an open access article distributed under the Creative Commons Attribution License, which permits unrestricted use, distribution, and reproduction in any medium, provided the original work is properly cited.

We deal with the existence and uniqueness of positive solutions to a class of nonlinear parabolic partial differential equations, by using some fixed point theorems for mixed monotone operators with perturbation.

\section{Introduction}

In this paper, we consider a class of nonlinear parabolic partial differential equations of the form

$$
\begin{gathered}
u_{t}-2 \Delta u=f(t, u, u)+g(t, u), \quad(x, t) \in \Omega \times(0, T), \\
u(x, t)=0, \quad(x, t) \in \Omega \times\{0\} \cup \partial \Omega \times(0, T),
\end{gathered}
$$

where $\Omega$ is a bounded smooth domain in $\mathbf{R}^{n}$ and $0<T<$ $+\infty$; we denote $Q_{T}=\Omega \times(0, T)$.

Problems related to nonlinear parabolic equations arise in many mathematical models of applied science, such as nuclear science, chemical reactions, heat transfer, population dynamics, and biological sciences, and have attracted a great deal of attention in the literature; see [1-6] and the references therein. In recent years, there are many results about existence, uniqueness, blowing-up, global existence, critical exponent, and other properties of the solution; see [4, 5, 7-12], among others. Some of the authors who investigated parabolic equations were using the method of upper and lower solutions; see [11], for example. Different from the works mentioned above, in the present paper, we will utilize some fixed point theorems for mixed monotone operators with perturbation to study the existence and uniqueness of positive solutions to the nonlinear parabolic partial differential equation (1).

With this context in mind, the outline of this paper is as follows. In Section 2 we will recall certain results from the theory of notations and results of monotone operators.
In Section 3, we will provide some conditions under which problem (1) will have a unique positive solution. Finally, in Section 4, we will provide an example, which explicates the applicability of our result.

\section{Preliminaries}

In this sequel, we present some basic concepts in ordered Banach spaces and two fixed point theorems which we will use later. For convenience, we suggest that one refers to [1316] for details.

Suppose that $(E,\|\cdot\|)$ is a real Banach space which is partially ordered by a cone $P \subset E$; that is, $x \leq y$ if and only if $y-x \in P$. If $x \leq y$ and $x \neq y$, then we denote $x<y$ or $y>x$. By $\theta$ we denote the zero element of $E$. Recall that a nonempty closed convex set $P \subset E$ is a cone if it satisfies (i) $x \in P, \lambda \geq 0 \Rightarrow \lambda x \in P$; (ii) $x \in P,-x \in P \Rightarrow x=\theta$. $P$ is called normal if there exists a constant $N>0$ such that, for all $x, y \in E, \theta \leq x \leq y$ implies that $\|x\| \leq N\|y\|$; in this case $N$ is called the normality constant of $P$. We say that an operator $A: E \rightarrow E$ is increasing (decreasing) if $x \leq y$ implies that $A x \leq A y(A x \geq A y)$. For all $x, y \in E$, the notation $x \sim y$ means that there exist $\lambda>0$ and $\mu>0$ such that $\lambda x \leq y \leq \mu x$. Clearly, $\sim$ is an equivalence relation. Given $h>\theta$ (i.e., $h \geq \theta$ and $h \neq \theta$ ), we denote by $P_{h}$ the set $P_{h}=\{x \in E \mid x \sim h\}$. It is easy to see that $P_{h} \subset P$.

Definition 1 (see $[13,14]) . A: P \times P \rightarrow P$ is said to be a mixed monotone operator if $A(x, y)$ is increasing in $x$ and 
decreasing in $y$; that is, $u_{i}, v_{i}(i=1,2) \in P, u_{1} \leq u_{2}, v_{1} \geq v_{2}$ imply that $A\left(u_{1}, v_{1}\right) \leq A\left(u_{2}, v_{2}\right)$. Element $x \in P$ is called a fixed point of $A$ if $A(x, x)=x$.

Definition 2. An operator $A: P \rightarrow P$ is said to be subhomogeneous if it satisfies $A(t x) \geq t A x, \forall t \in(0,1), x \in P$.

Definition 3. Let $D=P$ and let $\beta$ be a real number with $0 \leq$ $\beta<1$. An operator $A: D \rightarrow D$ is said to be $\beta$-concave if it satisfies $A(t x) \geq t^{\beta} A(x), \forall t \in(0,1), x \in D$.

Lemma 4 (see Theorem 2.1 in [16]). Let $\beta \in(0,1)$. $A$ : $P \times P \rightarrow P$ is a mixed monotone operator and satisfies $A\left(t x, t^{-1} y\right) \geq t^{\beta} A(x, y), \forall t \in(0,1), x, y \in P . B: P \rightarrow P$ is an increasing subhomogeneous operator. Assume that (i) there is $h_{0} \in P_{h}$ such that $A\left(h_{0}, h_{0}\right) \in P_{h}$ and $B h_{0} \in P_{h}$ and (ii) there exists a constant $\delta_{0}>0$ such that $A(x, y) \geq \delta_{0} B x, \forall x, y \in P$. Then we have the following:

(1) $A: P_{h} \times P_{h} \rightarrow P_{h}$ and $B: P_{h} \rightarrow P_{h}$;

(2) there exist $u_{0}, v_{0} \in P_{h}$ and $r \in(0,1)$ such that

$$
\begin{gathered}
r v_{0} \leq u_{0}<v_{0}, \\
u_{0} \leq A\left(u_{0}, v_{0}\right)+B u_{0} \leq A\left(v_{0}, u_{0}\right)+B v_{0} \leq v_{0}
\end{gathered}
$$

(3) the operator equation $A(x, x)+B x=x$ has a unique solution $x^{*}$ in $P_{h}$;

(4) for any initial values $x_{0}, y_{0} \in P_{h}$, constructing successively the sequences

$$
\begin{array}{r}
x_{n}=A\left(x_{n-1}, y_{n-1}\right)+B x_{n-1}, \\
y_{n}=A\left(y_{n-1}, x_{n-1}\right)+B y_{n-1}, \\
n=1,2, \ldots,
\end{array}
$$

one has $x_{n} \rightarrow x^{*}$ and $y_{n} \rightarrow x^{*}$ as $n \rightarrow \infty$.

Lemma 5 (see Theorem 2.4 in [16]). Let $\beta \in(0,1)$. $A$ : $P \times P \rightarrow P$ is a mixed monotone operator and satisfies $A\left(t x, t^{-1} y\right) \geq t A(x, y), \forall t \in(0,1), x, y \in P . B: P \rightarrow P$ is an increasing $\beta$-concave operator. Assume that (i) there is $h_{0} \in P_{h}$ such that $A\left(h_{0}, h_{0}\right) \in P_{h}$ and $B h_{0} \in P_{h}$ and (ii) there exists $a$ constant $\delta_{0}>0$ such that $A(x, y) \leq \delta_{0} B x, \forall x, y \in P$. Then we have the following:

(1) $A: P_{h} \times P_{h} \rightarrow P_{h}$ and $B: P_{h} \rightarrow P_{h}$;

(2) there exist $u_{0}, v_{0} \in P_{h}$ and $r \in(0,1)$ such that

$$
\begin{gathered}
r v_{0} \leq u_{0}<v_{0}, \\
u_{0} \leq A\left(u_{0}, v_{0}\right)+B u_{0} \leq A\left(v_{0}, u_{0}\right)+B v_{0} \leq v_{0}
\end{gathered}
$$

(3) the operator equation $A(x, x)+B x=x$ has a unique solution $x^{*}$ in $P_{h}$;
(4) for any initial values $x_{0}, y_{0} \in P_{h}$, constructing successively the sequences

$$
\begin{array}{r}
x_{n}=A\left(x_{n-1}, y_{n-1}\right)+B x_{n-1}, \\
y_{n}=A\left(y_{n-1}, x_{n-1}\right)+B y_{n-1}, \\
n=1,2, \ldots,
\end{array}
$$

one has $x_{n} \rightarrow x^{*}$ and $y_{n} \rightarrow x^{*}$ as $n \rightarrow \infty$.

\section{Existence and Uniqueness of Positive Solutions}

In this section, we will apply Lemmas 4 and 5 to study the problem (1), and we obtain some new results on the existence and uniqueness of positive solutions. The method used here is relatively new to the literature and so are the existence and uniqueness results of the nonlinear parabolic partial differential equations.

In our considerations, we work in the Banach space $E=$ $C\left(\overline{Q_{T}}\right)=\left\{u: \overline{Q_{T}} \rightarrow \mathbf{R}\right.$ is continuous $\}$ with the standard norm $\|u\|=\max \left\{|u(x, t)|:(x, t) \in \overline{Q_{T}}\right\}$. Notice that this space can be equipped with a partial order given by

$$
u, v \in E, \quad u \leq v \Longleftrightarrow u(x, t) \leq v(x, t), \quad(x, t) \in \overline{Q_{T}} .
$$

Set $P=\left\{u \in C\left(\overline{Q_{T}}\right) \mid u(x, t) \geq 0,(x, t) \in \overline{Q_{T}}\right\}$, as the standard cone. It is clear that $P$ is a normal cone in $C\left(\overline{Q_{T}}\right)$ and the normality constant is 1 .

The following assumptions on the nonlinear functions $f(x, u, v)$ and $g(x, u)$ are as follows:

$\left(\mathrm{H}_{1}\right) f:[0, T] \times[0,+\infty) \times[0,+\infty) \rightarrow[0,+\infty)$ is continuous and $g:[0, T] \times[0,+\infty) \rightarrow[0,+\infty)$ is continuous;

$\left(\mathrm{H}_{2}\right) f(t, u, v)$ is increasing in $u \in[0,+\infty)$, for fixed $t \in$ $[0, T]$ and $v \in[0,+\infty)$, and decreasing in $v \in[0,+\infty)$, for fixed $t \in[0, T]$ and $u \in[0,+\infty)$, and $g(t, u)$ is increasing in $u \in[0,+\infty)$, for fixed $t \in[0, T]$;

$\left(\mathrm{H}_{3}\right) g(t, \lambda u) \geq \lambda g(t, u)$, for $\lambda \in(0,1), t \in[0, T]$, and $u \in[0,+\infty)$, and there exists a constant $\beta \in(0,1)$ such that $f\left(t, \lambda u, \lambda^{-1} v\right) \geq \lambda^{\beta} f(t, u, v), \forall t \in[0, T]$, $\lambda \in(0,1), u, v \in[0,+\infty) ;$

$\left(\mathrm{H}_{4}\right)$ there exists a constant $\delta_{0}>0$ such that $f(t, u, v) \geq$ $\delta_{0} g(t, u), t \in[0, T], u, v \geq 0$;

$\left(\mathrm{H}_{5}\right) \min _{(x, t) \in \overline{\mathrm{Q}_{T}}} f(t, \varphi, \varphi)>0, \min _{(x, t) \in \overline{\mathrm{Q}_{T}}} g(t, \varphi)>0$, where $\varphi$ is the positive solution of the equation

$$
\begin{gathered}
u_{t}-\Delta u=1, \quad(x, t) \in \Omega \times(0, T), \\
u(x, t)=0, \quad(x, t) \in \Omega \times\{0\} \cup \partial \Omega \times(0, T) ;
\end{gathered}
$$

that is, $\varphi(x, t) \geq 0$ for $(x, t) \in \overline{Q_{T}}$. The existence of $\varphi$ follows from Theorem 7 in [17, Chapter 7].

Theorem 6. Assume that $\left(H_{1}\right)-\left(H_{5}\right)$ are satisfied. Then the nonlinear parabolic partial differential equation (1) has a unique positive solution $u^{*}$ in $P_{\varphi}$. 
Proof. We divide the proof into three steps.

Step 1. By the existence and uniqueness of linear parabolic partial differential equations, we consider the operator $A$ : $E \times E \rightarrow E$ as the solution of (8) for given $u, v \in P_{\varphi}$ as follows:

$$
\begin{aligned}
& \frac{\partial A(u, v)}{\partial t}-\Delta A(u, v)=f(t, u, v) \quad \text { in } Q_{T}, \\
& A(u, v)=0 \quad \text { on } \Omega \times\{0\} \cup \partial \Omega \times(0, T) .
\end{aligned}
$$

Indeed, for $t \in[0, T], u, v \in E$, we have $f(t, u, v) \in L^{n}\left(\overline{Q_{T}}\right)$. From the regularity theory of the heat equation, we conclude that $A(u, v) \in W^{2,1}\left(\overline{Q_{T}}\right)$, and there exists some $\alpha>0$ such that $W^{2,1}\left(\overline{Q_{T}}\right) \subset C^{2 \alpha, \alpha}\left(\overline{Q_{T}}\right)$, so the operator $A(u, v)$ is well defined as the solution of (8) for given $u, v \in P_{\varphi}$. For $u, v \in P_{\varphi}$, $\exists r_{u}, r_{v} \in(0,1)$ such that

$$
\begin{aligned}
& r_{u} \varphi(x, t) \leq u(x, t) \leq \frac{1}{r_{u}} \varphi(x, t), \\
& r_{v} \varphi(x, t) \leq v(x, t) \leq \frac{1}{r_{v}} \varphi(x, t),
\end{aligned}
$$

for $(x, t) \in \overline{Q_{T}}$. Let $r_{0}=\min \left\{r_{u}, r_{v}\right\}$. Then from $\left(\mathrm{H}_{3}\right)$,

$$
\begin{aligned}
f(t, u(x, t), v(x, t)) & \geq f\left(t, r_{0} \varphi(x, t), \frac{1}{r_{0}} \varphi(x, t)\right) \\
& \geq r_{0}^{\beta} f(t, \varphi(x, t), \varphi(x, t)), \\
f(t, u(x, t), v(x, t)) & \leq f\left(t, \frac{1}{r_{0}} \varphi(x, t), r_{0} \varphi(x, t)\right) \\
& \leq \frac{1}{r_{0}^{\beta}} f(t, \varphi(x, t), \varphi(x, t)),
\end{aligned}
$$

for $(x, t) \in \overline{Q_{T}}, u, v \in P_{\varphi}$. By $\left(\mathrm{H}_{3}\right)$ and the above inequalities, we have

$$
\begin{aligned}
& \frac{\partial A(u, v)}{\partial t}-\Delta A(u, v) \\
& =f(t, u, v) \geq r_{0}^{\beta} f(t, \varphi, \varphi) \geq r_{0}^{\beta} \min _{(x, t) \in \overline{Q_{T}}} f(t, \varphi, \varphi) \\
& =r_{0}^{\beta} \min _{(x, t) \in \overline{Q_{T}}} f(t, \varphi, \varphi)\left(\frac{\partial \varphi}{\partial t}-\Delta \varphi\right), \\
& \frac{\partial A(u, v)}{\partial t}-\Delta A(u, v) \\
& =f(t, u, v) \leq \frac{1}{r_{0}^{\beta}} f(t, \varphi, \varphi) \leq \frac{1}{r_{0}^{\beta}} \max _{(x, t) \in \overline{Q_{T}}} f(t, \varphi, \varphi) \\
& =\frac{1}{r_{0}^{\beta}} \max _{(x, t) \in \overline{Q_{T}}} f(t, \varphi, \varphi)\left(\frac{\partial \varphi}{\partial t}-\Delta \varphi\right),
\end{aligned}
$$

for $(x, t) \in Q_{T}, u, v \in P_{\varphi}$. By the comparison principle for parabolic partial differential equations $[18$, Lemma 3.1.8, page 108], we know that

$$
\frac{1}{r_{0}^{\beta}} \max _{(x, t) \in \overline{\mathrm{Q}_{T}}} f(t, \varphi, \varphi) \varphi \geq A(u, v) \geq r_{0}^{\beta} \min _{(x, t) \in \overline{\mathrm{Q}_{T}}} f(t, \varphi, \varphi) \varphi \geq 0,
$$

for $u, v \in P_{\varphi}$, which is satisfied by $\left(\mathrm{H}_{5}\right)$. So $A: P_{\varphi} \times P_{\varphi} \rightarrow P$.

Let $r_{1}=r_{0}^{\beta} \min _{(x, t) \in \overline{Q_{T}}} f(t, \varphi, \varphi)$ and $r_{2}=\left(1 / r_{0}^{\beta}\right)$ $\max _{(x, t) \in \overline{\mathrm{Q}_{T}}} f(t, \varphi, \varphi)$; we get that $r_{2} \varphi \geq A(u, v) \geq r_{1} \varphi$, for $u, v \in P_{\varphi}$; that is, $A(\varphi, \varphi) \in P_{\varphi}$. By $\left(\mathrm{H}_{2}\right)$, we have

$$
\begin{gathered}
\frac{\partial A_{u}(u, v)}{\partial t}-\Delta A_{u}(u, v)=f_{u}(t, u, v) \geq 0, \\
\frac{\partial A_{v}(u, v)}{\partial t}-\Delta A_{v}(u, v)=f_{v}(t, u, v) \leq 0,
\end{gathered}
$$

for $(x, t) \in Q_{T}, u, v \in P_{\varphi}$. Also, by the comparison principle for parabolic partial differential equations, we observe that $A_{u} \geq 0, A_{v} \leq 0$; that is, $A$ is a mixed monotone operator. Moreover, by $\left(\mathrm{H}_{3}\right)$, we have

$$
\begin{gathered}
\frac{\partial A\left(\lambda u, \lambda^{-1} v\right)}{\partial t}-\Delta A\left(\lambda u, \lambda^{-1} v\right) \\
=f\left(t, \lambda u, \lambda^{-1} v\right) \geq \lambda^{\beta} f(t, u, v) \\
=\frac{\partial\left(\lambda^{\beta} A(u, v)\right)}{\partial t}-\Delta\left(\lambda^{\beta} A(u, v)\right),
\end{gathered}
$$

for $(x, t) \in Q_{T}, u, v \in P_{\varphi}$. Also, by the comparison principle for parabolic partial differential equations, we conclude that $A\left(\lambda u, \lambda^{-1} v\right) \geq \lambda^{\beta} A(u, v)$, for $u, v \in P_{\varphi}$.

Step 2. Similar to Step 1, we can also construct the operator $B: E \rightarrow E$ as the solution of (15) for given $u \in P_{\varphi}$ :

$$
\begin{gathered}
\frac{\partial B u}{\partial t}-\Delta B u=g(t, u) \quad \text { in } Q_{T}, \\
B u=0 \quad \text { on } \Omega \times\{0\} \cup \partial \Omega \times(0, T),
\end{gathered}
$$

and we get the fact that the operator $B u$ is well defined as the solution of (15) for given $u \in P_{\varphi}$. From $\left(\mathrm{H}_{3}\right)$, we get

$$
\begin{aligned}
& g(t, u(x, t)) \geq g\left(t, r_{0} \varphi(x, t)\right) \geq r_{0} g(t, \varphi(x, t)), \\
& g(t, u(x, t)) \leq g\left(t, \frac{1}{r_{0}} \varphi(x, t)\right) \leq \frac{1}{r_{0}} g(t, \varphi(x, t)),
\end{aligned}
$$


for $(x, t) \in \overline{Q_{T}}, u \in P_{\varphi}$. By $\left(\mathrm{H}_{3}\right)$ and the above inequalities, we have

$$
\begin{aligned}
\frac{\partial B u}{\partial t}-\Delta B u & =g(t, u) \\
& \geq r_{0} g(t, \varphi) \geq r_{0} \min _{(x, t) \in \overline{\mathrm{Q}_{T}}} g(t, \varphi) \\
& =r_{0} \min _{(x, t) \in \overline{\mathrm{Q}_{T}}} g(t, \varphi)\left(\frac{\partial \varphi}{\partial t}-\Delta \varphi\right), \\
\frac{\partial B u}{\partial t}-\Delta B u & =g(t, u) \\
& \leq \frac{1}{r_{0}} g(t, \varphi) \leq \frac{1}{r_{0}} \max _{(x, t) \in \overline{\mathrm{Q}_{T}}} g(t, \varphi) \\
& =\frac{1}{r_{0}} \max _{(x, t) \in \overline{\mathrm{Q}_{T}}} g(t, \varphi)\left(\frac{\partial \varphi}{\partial t}-\Delta \varphi\right),
\end{aligned}
$$

for $(x, t) \in Q_{T}, u \in P_{\varphi}$. Also, by the comparison principle for parabolic partial differential equations, we know that

$$
\frac{1}{r_{0}} \max _{(x, t) \in \overline{\mathrm{Q}_{T}}} g(t, \varphi) \varphi \geq B u \geq r_{0} \min _{(x, t) \in \overline{\mathrm{Q}_{T}}} g(t, \varphi) \varphi \geq 0,
$$

for $u \in P_{\varphi}$, which is satisfied by $\left(\mathrm{H}_{5}\right)$. So $B: P_{\varphi} \rightarrow P$.

Let $r_{3}=r_{0} \min _{(x, t) \in \overline{\mathrm{Q}_{T}}} g(t, \varphi)$ and $r_{4}=\left(1 / r_{0}\right)$ $\max _{(x, t) \in \overline{Q_{T}}} g(t, \varphi)$; we get that $r_{4} \varphi \geq B u \geq r_{3} \varphi$, where $u \in P_{\varphi}$; that is, $B \varphi \in P_{\varphi}$. By $\left(\mathrm{H}_{2}\right)$, we have

$$
\frac{\partial B_{u} u}{\partial t}-\Delta B_{u} u=g_{u}(t, u) \geq 0
$$

for $(x, t) \in Q_{T}, u \in P_{\varphi}$. By the comparison principle for parabolic partial differential equations, we observe that $B_{u} \geq$ 0 ; that is, $B$ is an increasing operator. Moreover, by $\left(\mathrm{H}_{3}\right)$, we have

$$
\begin{aligned}
\frac{\partial B(\lambda u)}{\partial t}-\Delta B(\lambda u) & =g(t, \lambda u) \geq \lambda g(t, u) \\
& =\frac{\partial(\lambda B u)}{\partial t}-\Delta(\lambda B u)
\end{aligned}
$$

for $(x, t) \in Q_{T}, u \in P_{\varphi}$. By the comparison principle for parabolic partial differential equations, we conclude that $B(\lambda u) \geq \lambda B u$, for $u \in P_{\varphi}$.

Step 3. By $\left(\mathrm{H}_{4}\right)$, we have

$$
\begin{aligned}
\frac{\partial A(u, v)}{\partial t}-\Delta A(u, v) & =f(t, u, v) \geq \delta_{0} g(t, u) \\
& =\frac{\partial\left(\delta_{0} B u\right)}{\partial t}-\Delta\left(\delta_{0} B u\right),
\end{aligned}
$$

for $(x, t) \in Q_{T}, u, v \in P_{\varphi}$. By the comparison principle for parabolic partial differential equations, we know that $A(u, v) \geq \delta_{0} B u$, where $u, v \in P_{\varphi}$.
Therefore, the operators $A$ and $B$ satisfy all the conditions in Lemma 4. Thus, the operator equation $A(u, u)+B u=u$ has a unique solution $u^{*}$ in $P_{\varphi}$. By

$$
\begin{aligned}
& \frac{\partial u^{*}}{\partial t}= \frac{\partial A\left(u^{*}, u^{*}\right)}{\partial t}+\frac{\partial B u^{*}}{\partial t} \\
&=\left(\Delta A\left(u^{*}, u^{*}\right)+f\left(t, u^{*}, u^{*}\right)\right)+\left(\Delta B u^{*}+g\left(t, u^{*}\right)\right) \\
&= 2 \Delta u^{*}+f\left(t, u^{*}, u^{*}\right)+g\left(t, u^{*}\right) \\
&(x, t) \in \Omega \times(0, T) \\
& u^{*}=A\left(u^{*}, u^{*}\right)+B u^{*}=0 \\
&(x, t) \in \Omega \times\{0\} \cup \partial \Omega \times(0, T)
\end{aligned}
$$

we obtain that the nonlinear parabolic partial differential equation (1) has a unique positive solution $u^{*}$ in $P_{\varphi}$. The proof is complete.

Further, we make some other assumptions on the nonlinear functions $f(t, u, v)$ and $g(t, u)$ :

$\left(\mathrm{H}_{6}\right)$ there exists a constant $\beta \in(0,1)$ such that $g(t, \lambda u) \geq$ $\lambda^{\beta} g(t, u), \forall t \in[0, T], \lambda \in(0,1), u \in[0,+\infty)$, and $f\left(t, \lambda u, \lambda^{-1} v\right) \geq \lambda f(t, u, v)$, for $\lambda \in(0,1), t \in[0, T]$, $u, v \in[0,+\infty)$;

$\left(\mathrm{H}_{7}\right)$ there exists a constant $\delta_{0}>0$ such that $f(t, u, v) \leq$ $\delta_{0} g(t, u), t \in[0, T], u, v \geq 0$.

By using Lemma 5, we can also easily prove the following conclusion.

Theorem 7. Assume that $\left(\mathrm{H}_{1}\right),\left(\mathrm{H}_{2}\right),\left(\mathrm{H}_{5}\right),\left(\mathrm{H}_{6}\right)$, and $\left(\mathrm{H}_{7}\right)$ are satisfied. Then the nonlinear parabolic partial differential equation (1) has a unique positive solution $u^{*}$ in $P_{\varphi}$.

\section{An Example}

We now present one example to illustrate Theorem 6.

Example 1. Consider the following parabolic partial differential equation:

$$
\begin{array}{r}
u_{t}-2 \Delta u=u^{1 / 2}+(u+1)^{-1 / 3}+\frac{u}{u+1} a(t) \\
+b(t)+c, \quad(x, t) \in \Omega \times(0,1), \\
u(x, t)=0, \quad(x, t) \in \Omega \times\{0\} \cup \partial \Omega \times(0,1),
\end{array}
$$

where $c>0$ is a constant, $a, b:[0,1] \rightarrow(0,+\infty)$ are continuous, and $\Omega$ is a bounded smooth domain in $\mathbf{R}^{n}$; we denote $Q_{1}=\Omega \times(0,1)$. Set $\varphi$ to be the positive solution of the following:

$$
\begin{gathered}
u_{t}-\Delta u=1, \quad(x, t) \in \Omega \times(0,1), \\
u(x, t)=0, \quad(x, t) \in \Omega \times\{0\} \cup \partial \Omega \times(0,1) ;
\end{gathered}
$$


that is, $\varphi(x, t) \geq 0$, for $(x, t) \in \overline{Q_{1}}$. We can conclude that the nonlinear parabolic partial differential equation (23) has a unique positive solution $u^{*}$ in $P_{\varphi}$.

Proof. In this example, we take $0<d<c$ and let

$$
\begin{gathered}
f(t, u, v)=u^{1 / 2}+(v+1)^{-1 / 3}+b(t)+d, \\
g(t, u)=\frac{u}{u+1} a(t)+c-d, \\
\beta=\frac{1}{2}, \quad a^{*}=\max \{a(t): t \in[0,1]\} .
\end{gathered}
$$

Obviously, $a^{*}>0, f:[0,1] \times[0,+\infty) \times[0,+\infty) \rightarrow[0,+\infty)$ is continuous, and $g:[0,1] \times[0,+\infty) \rightarrow[0,+\infty)$ is continuous. And $f(t, u, v)$ is increasing in $u \in[0,+\infty)$, for fixed $t \in[0,1]$ and $v \in[0,+\infty)$, and decreasing in $v \in$ $[0,+\infty)$, for fixed $t \in[0,1]$ and $u \in[0,+\infty)$, and $g(t, u)$ is increasing in $u \in[0,+\infty)$, for fixed $t \in[0,1]$. Besides, for $\lambda \in(0,1), t \in[0,1]$, and $u, v \in[0,+\infty)$, we have

$$
\begin{aligned}
g(t, \lambda u) & =\frac{\lambda u}{\lambda u+1} a(t)+c-d \\
& \geq \frac{\lambda u}{u+1} a(t)+\lambda(c-d)=\lambda g(t, u), \\
f\left(t, \lambda u, \lambda^{-1} v\right) & =(\lambda u)^{1 / 2}+\left(\lambda^{-1} v+1\right)^{-1 / 3}+b(t)+d \\
& \geq \lambda^{1 / 2}\left[u^{1 / 2}+(v+1)^{-1 / 3}+b(t)+d\right] \\
& =\lambda^{1 / 2} f(t, u, v) .
\end{aligned}
$$

Moreover, if we take $\delta_{0} \in\left(0, d /\left(a^{*}+c-d\right)\right]$, then we obtain

$$
\begin{aligned}
f(t, u, v) & =u^{1 / 2}+(v+1)^{-1 / 3}+b(t)+d \geq d \\
& =\frac{d}{a^{*}+c-d} \cdot\left(a^{*}+c-d\right) \\
& \geq \delta_{0}\left[\frac{u}{u+1} a(t)+c-d\right]=\delta_{0} g(t, u) .
\end{aligned}
$$

Further, $\min _{(x, t) \in \overline{\mathrm{Q}_{1}}} f(t, \varphi, \varphi) \geq d>0$ and $\min _{(x, t) \in \overline{\mathrm{Q}_{1}}} g(t, \varphi) \geq$ $c-d>0$.

Hence, all the conditions of Theorem 6 are satisfied. An application of Theorem 6 implies that the nonlinear parabolic partial differential equation (23) has a unique positive solution $u^{*}$ in $P_{\varphi}$, where $P_{\varphi}=\left\{u \in C\left(\overline{Q_{1}}\right) \mid\right.$ there exist $\lambda=$ $\lambda(u, \varphi), \mu=\mu(u, \varphi)>0$ such that $\lambda \varphi \leq u \leq \mu \varphi\}$.

\section{Conflict of Interests}

The authors declare that there is no conflict of interests regarding the publication of this paper.

\section{Authors' Contribution}

The authors declare that the study was realized in collaboration with the same responsibility. All authors read and approved the final paper.

\section{Acknowledgments}

The authors are grateful to the anonymous referee for his/her valuable suggestions. This paper was supported financially by the Youth Science Foundations of China (11201272) and Shanxi Province (2010021002-1). The second author was partially supported by Shanxi Scholarship Council of China.

\section{References}

[1] E. Acerbi and G. Mingione, "Regularity results for stationary electro-rheological fluids," Archive for Rational Mechanics and Analysis, vol. 164, no. 3, pp. 213-259, 2002.

[2] S. Antontsev and S. Shmarev, "Blow-up of solutions to parabolic equations with nonstandard growth conditions," Journal of Computational and Applied Mathematics, vol. 234, no. 9, pp. 2633-2645, 2010.

[3] L. Diening, P. Harjulehto, P. Hästö, and M. Ruzicka, Lebesgue and Sobolev Spaces with Variable Exponents, vol. 2017 of Lecture Notes in Mathematics, Springer, Heidelberg, Germany, 2011.

[4] B. Hu and H.-M. Yin, "Semilinear parabolic equations with prescribed energy," Rendiconti del Circolo Matematico di Palermo. Serie II, vol. 44, no. 3, pp. 479-505, 1995.

[5] J. P. Pinasco, "Blow-up for parabolic and hyperbolic problems with variable exponents," Nonlinear Analysis: Theory, Methods \& Applications, vol. 71, no. 3-4, pp. 1094-1099, 2009.

[6] P. Quittner and P. Souplet, Superlinear Parabolic Problems. BlowUp, Global Existence and Steady States, Birkhauser Advanced Texts, Berlin, Germany, 2007.

[7] R. Ferreira, A. de Pablo, M. Pérez-LLanos, and J. D. Rossi, "Critical exponents for a semilinear parabolic equation with variable reaction," Proceedings of the Royal Society of Edinburgh. Section A. Mathematics, vol. 142, no. 5, pp. 1027-1042, 2012.

[8] W. Gao and Y. Han, "Blow-up of a nonlocal semilinear parabolic equation with positive initial energy," Applied Mathematics Letters, vol. 24, no. 5, pp. 784-788, 2011.

[9] M. Ishiwata and T. Suzuki, "Positive solution to semilinear parabolic equation associated with critical Sobolev exponent," Nonlinear Differential Equations and Applications, vol. 20, no. 4, pp. 1553-1576, 2013.

[10] W. Liu and M. Wang, "Blow-up of the solution for a $p$-Laplacian equation with positive initial energy," Acta Applicandae Mathematicae, vol. 103, no. 2, pp. 141-146, 2008.

[11] C. V. Pao and W. H. Ruan, "Positive solutions of quasilinear parabolic systems with Dirichlet boundary condition," Journal of Differential Equations, vol. 248, no. 5, pp. 1175-1211, 2010.

[12] X. Wu, B. Guo, and W. Gao, "Blow-up of solutions for a semilinear parabolic equation involving variable source and positive initial energy," Applied Mathematics Letters, vol. 26, no. 5, pp. 539-543, 2013.

[13] D. J. Guo and V. Lakshmikantham, "Coupled fixed points of nonlinear operators with applications," Nonlinear Analysis: Theory, Methods \& Applications, vol. 11, no. 5, pp. 623-632, 1987.

[14] D. J. Guo, "Fixed points of mixed monotone operators with applications," Applicable Analysis, vol. 31, no. 3, pp. 215-224, 1988.

[15] C. Zhai and L. Zhang, "New fixed point theorems for mixed monotone operators and local existence-uniqueness of positive solutions for nonlinear boundary value problems," Journal of Mathematical Analysis and Applications, vol. 382, no. 2, pp. 594614, 2011. 
[16] C. Zhai and M. Hao, "Fixed point theorems for mixed monotone operators with perturbation and applications to fractional differential equation boundary value problems," Nonlinear Analysis: Theory, Methods and Applications, vol. 75, no. 4, pp. 25422551, 2012.

[17] L. C. Evans, Partial Differential Equations, American Mathematical Society, 1998.

[18] Q. X. Ye and Z. Y. Li, Introduction of Reaction-Diffusion Equations, Science Press, Beijing, China, 1994, (Chinese). 


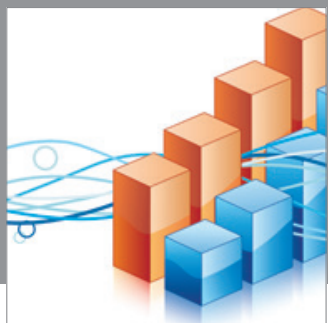

Advances in

Operations Research

mansans

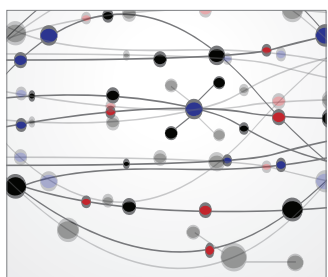

The Scientific World Journal
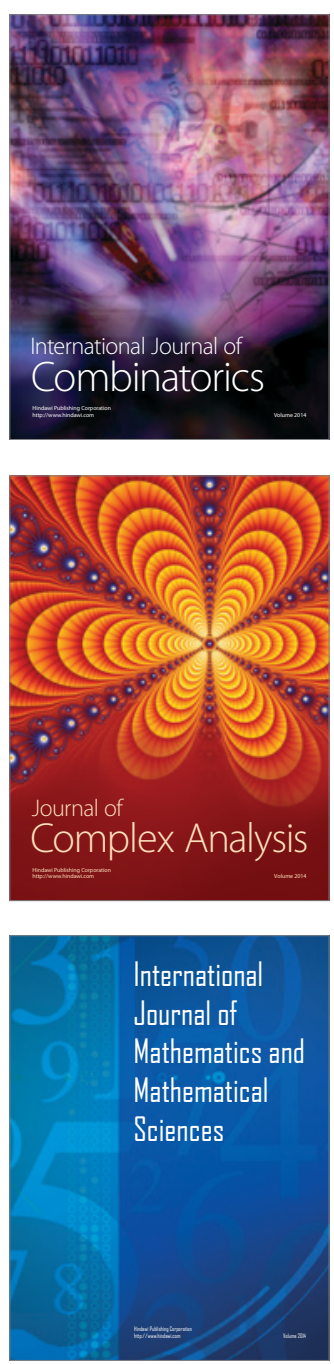
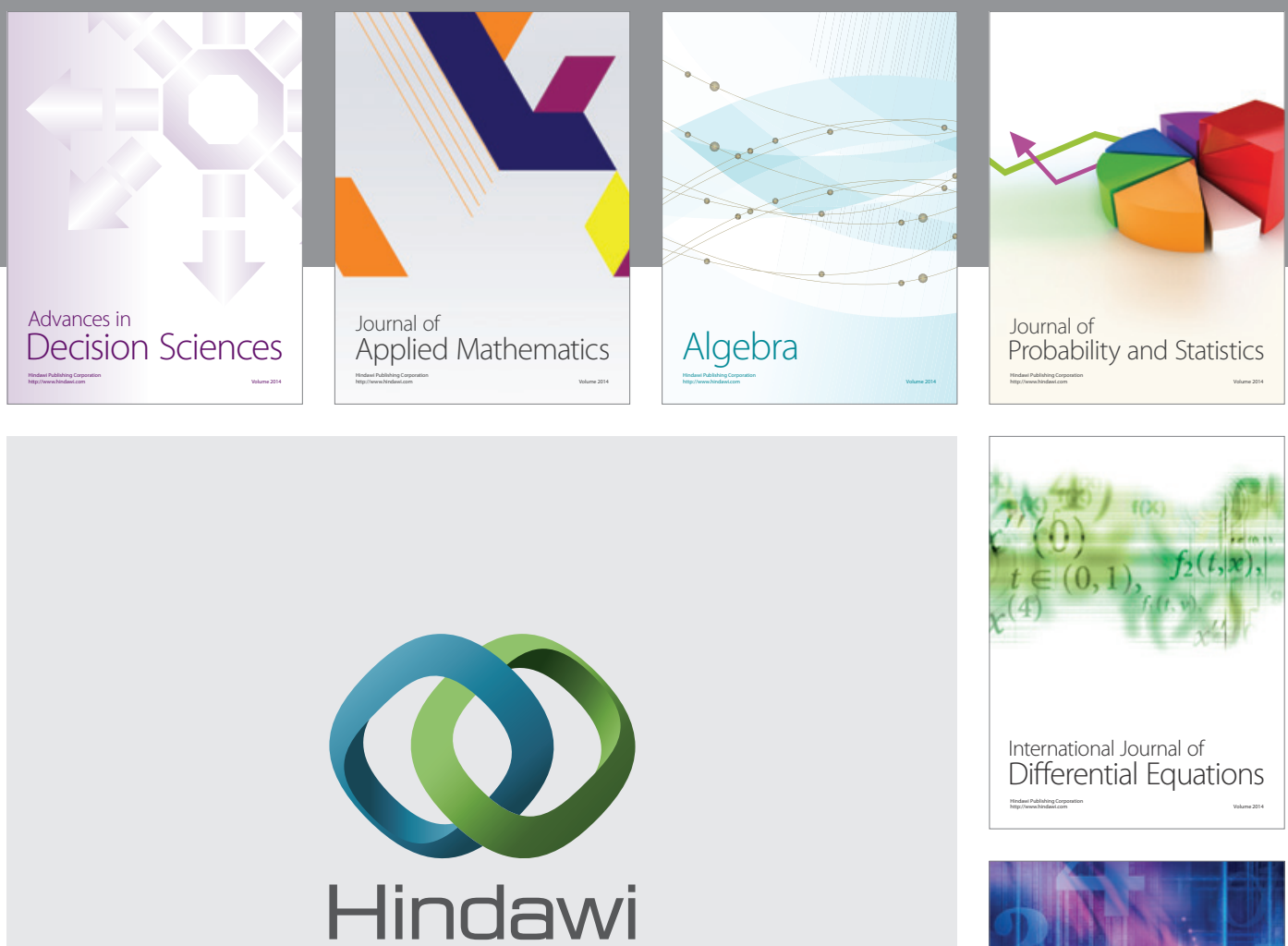

Submit your manuscripts at http://www.hindawi.com
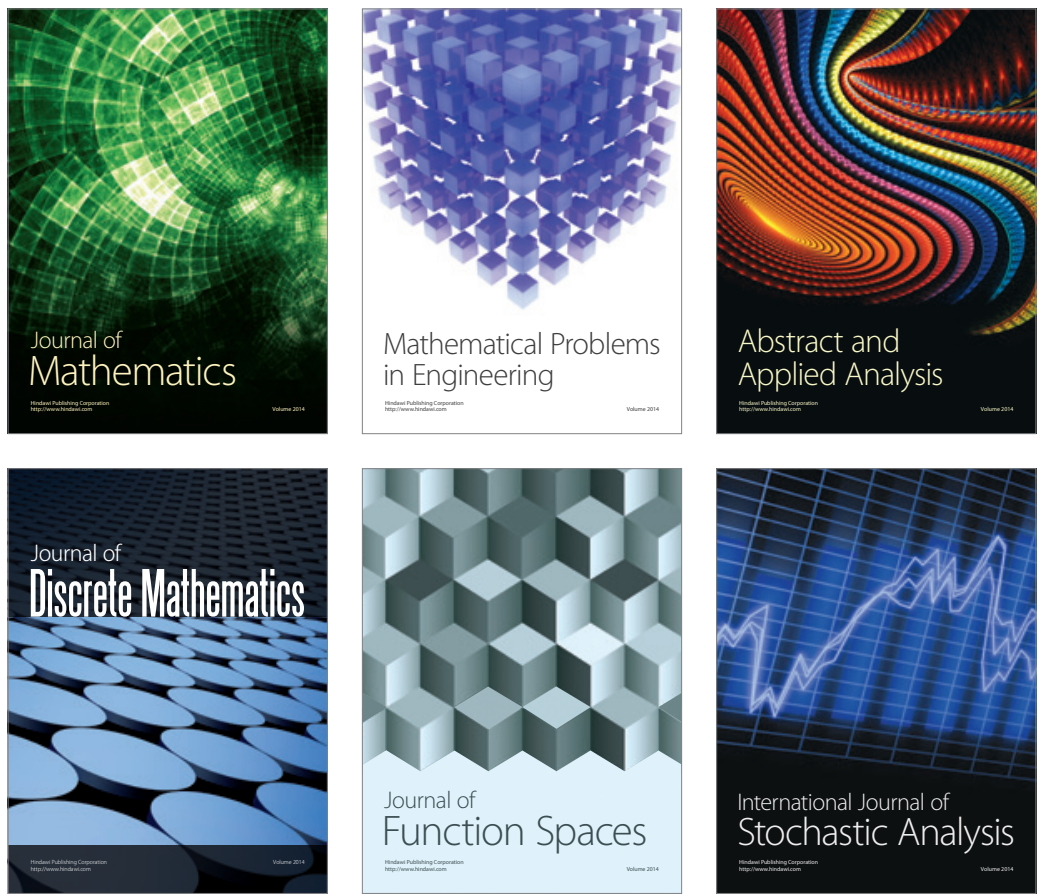

Journal of

Function Spaces

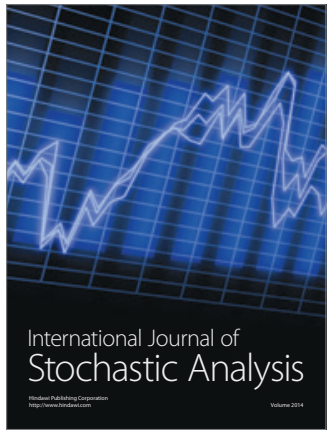

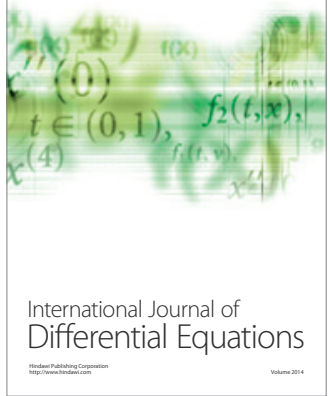
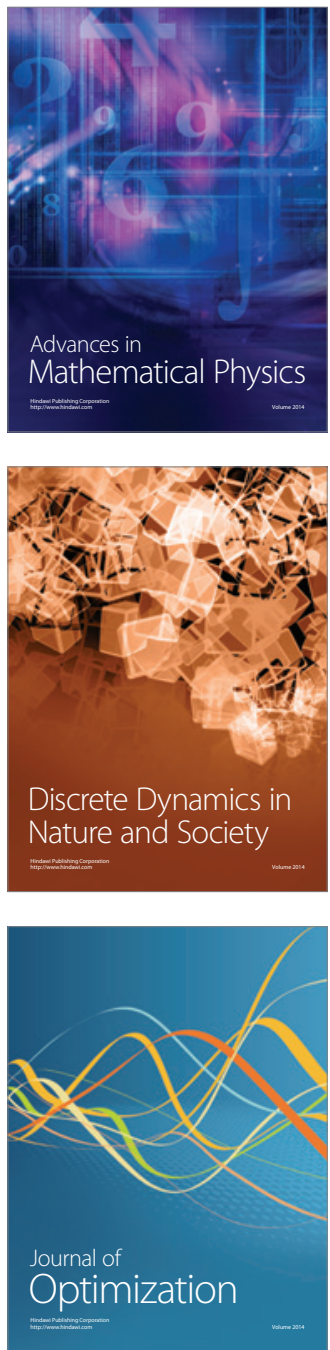